\title{
青藏高原高寒草地3米深度土壤无机碳库及分布特征
}

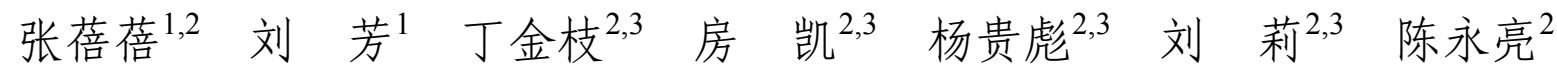 \\ 李飞 2,3 杨元合 ${ }^{2}$
}

${ }^{1}$ 内蒙古工业大学能源与动力工程学院, 呼和浩特 $010051{ }^{2}$ 中国科学院植物研究所植被与环境变化国家重点实验室, 北京 $100093 ;{ }^{3}$ 中国科学院大学, 北京 100049

摘 要 准确评估土壤无机碳库的大小及其分布特征有助于全面理解陆地生态系统碳循环与气候变暖之间的反馈关系。然 而, 由于深层土壤剖面信息贯乏, 使得目前学术界对深层土壤无机碳库的了解十分有限。该研究基于 342 个 $3 \mathrm{~m}$ 深度和 177 个 50 $\mathrm{cm}$ 深度的土壤剖面信息, 采用克里格插值方法估算了青藏高原高寒草地不同深度的土壤无机碳库大小, 并在此基础上分析 了该地区土壤无机碳密度的分布特征。结果显示, 青藏高原高寒草地 $0-50 \mathrm{~cm} 、 0-1 \mathrm{~m} 、 0-2 \mathrm{~m}$ 和0-3 m深度的土壤无机碳库大 小分别为8.26、17.82、36.33和54.29 Pg C, 对应的土壤无机碳密度分别为7.22、15.58、31.76和47.46 $\mathrm{kg} \mathrm{C} \cdot \mathrm{m}^{-2}$ 。研究区土壤无 机碳密度总体呈现由东南向西北增加的趋势; 高寒草原土壤的无机碳密度显著大于高寒草甸的无机碳密度。整体上，不同深 度的高寒草原无机碳库约占整个研究区无机碳库的 $63 \%-66 \%$ 。此外, 深层土壤中储存了大量无机碳, $1 \mathrm{~m}$ 以下土壤无机碳库是 $1 \mathrm{~m}$ 以内无机碳库的2倍。两种草地类型土壤无机碳的垂直分布存在差异: 对高寒草原而言, 0-50 $\mathrm{cm}$ 土壤无机碳所占的比例最 大; 但对高寒草甸而言, 在100-150 cm深度土壤无机碳出现富集。这些结果表明青藏高原深层土壤是一个重要的无机碳库, 需在未来碳循环研究中予以重视。

关键词 碳库; 克里格插值; 土壤无机碳; $3 \mathrm{~m}$ 土钻; 青藏高原

引用格式: 张蓓蓓, 刘芳, 丁金枝, 房凯, 杨贵彪, 刘莉, 陈永亮, 李飞, 杨元合 (2016). 青藏高原高寒草地 3 米深度土壤无机碳库及分布特征. 植物生 态学报, 40, 93-101. doi: 10.17521/cjpe.2015.0406

\section{Soil inorganic carbon stock in alpine grasslands on the Qinghai-Xizang Plateau: An updated evaluation using deep cores}

ZHANG Bei-Bei ${ }^{1,2}$, LIU Fang ${ }^{1}$, DING Jin-Zhi ${ }^{2,3}$, FANG Kai ${ }^{2,3}$, YANG Gui-Biao ${ }^{2,3}$, LIU Li ${ }^{2,3}$, CHEN Yong-Liang ${ }^{2}$, LI Fei ${ }^{2,3}$, and YANG Yuan-He ${ }^{2 *}$

${ }^{1}$ College of Energy and Power Engineering, Inner Mongolia University of Technology, Hohhot 010051, China; ${ }^{2}$ State Key Laboratory of Vegetation and Environmental Change, Institute of Botany, Chinese Academy of Sciences, Beijing 100093, China; and ${ }^{3}$ University of Chinese Academy of Sciences, Beijing 100049, China

\section{Abstract}

Aims To estimate the size and spatial patterns of 3-m-deep soil inorganic carbon (SIC) stock across alpine grasslands on the Qinghai-Xizang Plateau.

Methods We conducted a comprehensive investigation and collected soil samples from 342 3-m-deep cores and 177 50-cm-deep pits across the study area. Using Kriging interpolation, we interpolated site-level observations to the regional level. The distribution of SIC density was then overlaid with the regional vegetation map at a scale of 1:1 000000 to calculate SIC stock of the alpine steppe and alpine meadow. Kruskal-Wallis tests were further conducted to examine the differences of SIC density between the two grassland types and among soil depths with 50 cm-depth intervals.

Important findings The total SIC stock at depths of $50 \mathrm{~cm}, 1 \mathrm{~m}, 2 \mathrm{~m}$ and $3 \mathrm{~m}$ were estimated at $8.26,17.82$, 36.33 and $54.29 \mathrm{Pg} \mathrm{C}$, with SIC density being $7.22,15.58,31.76$ and $47.46 \mathrm{~kg} \mathrm{C} \cdot \mathrm{m}^{-2}$, respectively. SIC density exhibited large spatial variability, with an increasing trend from the southeastern to the northwestern plateau. Much larger SIC stock was observed in the alpine steppe than alpine meadow, with the former accounting for $63 \%-66 \%$ of the total stock at depths of $50 \mathrm{~cm}, 1 \mathrm{~m}, 2 \mathrm{~m}$ and $3 \mathrm{~m}$. A large amount of SIC stock was found in deep soils (1-3 m), amounting to approximately 2 times as much carbon stored in the top 1-m-deep soil layer. The vertical distributions of SIC density differed between the two grassland types. The highest proportions of SIC

收稿日期Received: 2015-11-13 接受日期Accepted: 2016-01-17

* 通信作者Author for correspondence (E-mail: yhyang@ibcas.ac.cn) 
occurred in the upper $50 \mathrm{~cm}$ layer for the alpine steppe while the highest proportions occurred in $100-150 \mathrm{~cm}$ layer for the alpine meadow. These results highlight that a large amount of SIC is stored in deep soil layers, which should be considered in evaluating terrestrial carbon balance under global change scenario.

Key words carbon stock; Kriging interpolation; soil inorganic carbon; deep cores; Qinghai-Xizang Plateau

Citation: Zhang BB, Liu F, Ding JZ, Fang K, Yang GB, Liu L, Chen YL, Li F, Yang YH (2016). Soil inorganic carbon stock in alpine grasslands on the Qinghai-Xizang Plateau: An updated evaluation using deep cores. Chinese Journal of Plant Ecology, 40, 93-101. doi: 10.17521/cjpe.2015.0406

土壤无机碳在陆地碳循环中的作用不容忽视 (Mikhailova \& Post, 2006; Georg et al., 2008; Stone, 2008; 许乃政等, 2009; Zhang et al., 2015)。尤其在干 旱和半干旱地区, 土壤无机碳库比有机碳库大 $2-10$ 倍(Schlesinger, 1982; Somebroek, 1993; 杨黎芳等, 2006; 余健等, 2014), 可能在区域碳循环中扮演着 重要角色。一方面, 成土风化过程中次生碳酸盐的 形成可以固定大气或土壤中分解产生的 $\mathrm{CO}_{2}(\mathrm{Lal}$, 2004; Mi et al., 2008; Tan et al., 2014), 且生成的碳 酸盐较为稳定(Landi et al., 2003)。因此, 土壤无机碳 在减少大气 $\mathrm{CO}_{2}$ 浓度方面的贡献不容忽视(Jacobson et al., 2002; Lerman \& Mackenzie, 2005; Liu et al., 2010, 2011; 郑聚锋等, 2011)。另一方面, 工业革命 以来, 持续的大气氮沉降和农业活动引起的土壤酸 化导致了土壤中的大量碳酸盐以 $\mathrm{CO}_{2}$ 的形式释放到 大气中(Yang et al., 2010; Tan et al., 2014), 进而对 大气 $\mathrm{CO}_{2}$ 浓度产生重要影响。因此, 准确评估土壤无 机碳库的大小及其分布特征将加深学术界对土壤碳 循环特征的理解, 并有助于全面认识陆地生态系统 碳循环与气候变暖之间的反馈关系(Batjes, 1996; Harrison \& Dorn, 2014)。

青藏高原高寒草地总面积约为 $1.1 \times 10^{6} \mathrm{~km}^{2}$, 约占我国国土面积的 $12 \%$ 。更为重要的是, 青藏高 原高寒草地分布于我国典型的干旱、半干旱地区, 土壤无机碳密度大(Mi et al., 2008)。因此, 准确估算 青藏高原土壤无机碳库的大小是全面评估我国土壤 无机碳库、深入理解区域乃至国家尺度陆地碳循环 特征的基础。近年来, 已有一些研究报道了青藏高 原 1 m深度土壤无机碳库的大小(Yang et al., 2010; 刘淑丽等, 2014), 但对青藏高原深层土壤无机碳库 的研究还相对薄弱。而最近的一些研究表明, 在干 旱、半干旱地区, 深层土壤中的无机碳含量是浅层 土壤的 2 倍以上, 且在深层土壤剖面中土壤碳主要 以无机碳的形式存在(Díaz-Hernández, 2010; Wang et al., 2010), 因此, 限于 $1 \mathrm{~m}$ 以内的估算将严重低估
土壤无机碳库大小(Díaz-Hernández et al., 2003; Díaz-Hernández, 2010; Wang et al., 2010; 王海荣和 杨忠芳, 2011)。

为此, 本研究基于 342 个 $3 \mathrm{~m}$ 深度及 177 个 $50 \mathrm{~cm}$ 深度的土壤剖面样品, 利用克里格插值, 估算了青 藏高原高寒草地 $3 \mathrm{~m}$ 深度土壤无机碳库大小, 在此 基础上分析了青藏高原高寒草地土壤无机碳密度的 空间分布特征, 旨在为我国土壤无机碳库研究提供 数据支持。

\section{1 材料和方法}

\section{1 研究区域概况}

研究区分布于青藏高原, 平均海拔4 $000 \mathrm{~m}$ 以 上。该区气候寒冷干燥, 年平均气温为-3.09-4.43 ${ }^{\circ} \mathrm{C}$, 年降水量为103-694 mm (Yang et al., 2015)。其 中, $80 \%-90 \%$ 的降水量集中在 5 月到 9 月, 在空间上 呈现出沿东南到西北依次减少的规律(Yang et al., 2008)。研究区成土母质以冰碛物、残积-坡积物为 主(于伯华和吕昌河, 2011), 主要的土壤类型包括高 寒草原土和高寒草甸土(周兴民等，1986; 李明森, 1994)。高寒草地是青藏高原主要的植被类型, 主要 包括高寒草原及高寒草甸两类。高寒草原主要分布 在青藏高原西北部, 而高寒草甸主要分布在青藏高 原东南部。高寒草原分布区相对干旱, 年降水量为 $296 \mathrm{~mm}$, 优势种多数为旱生植物, 包括紫花针茅 (Stipa purpurea)、青藏薹草(Carex moorcroftii)、珠芽 苶(Polygonum viviparum), 以及一些高寒杂草, 如 龙胆属(Gentiana)、马先蒿属(Pedicularis)植物; 而高 寒草甸分布区相对湿润, 年降水量为 $460 \mathrm{~mm}$, 优势 种主要包括高山嵩草(Kobresia pygmaea)、矮生嵩草 (Kobresia humilis)及西藏嵩草(Kobresia tibetica)等 (Yang et al., 2010)。

\section{2 土壤样品采集}

为了估算青藏高原高寒草地 $3 \mathrm{~m}$ 深度土壤无机 碳库大小, 我们于 2013年和2014年的7-8月在研究 
区调查了 173 处样地, 相邻样地间平均直线距离约 为 $30 \mathrm{~km}$, 获取了共计 519 个土壤剖面样品(Ding et al., 2016)。这些调查样地基本覆盖了研究区主要的 气候类型、草地类型和土壤类型。其中, 91 个来自高 寒草原, 82 个来自高寒草甸。在每处样地, 设置 $10 \mathrm{~m}$ $\times 10 \mathrm{~m}$ 的大样方, 沿大样方的四角以及中心位置设 置 5 个 $1 \mathrm{~m} \times 1 \mathrm{~m}$ 的小样方。随后, 在大样方其中一条 对角线的 3 个小样方的中心位置获取不同层次的土 壤样品。在所调查的 173 个样地中, 有 114 个样地共 计 342 个土壤剖面使用机械钻取样, 取样深度分别 为 $0-10 、 10-20 、 20-30 、 30-50 、 50-70 、 70-100$ 、 100-150、150-200、200-250和250-300 cm。由于青 藏高原西部恶劣的气候条件和交通状况等客观因素 的限制, 深层土壤采样难以在西部实施, 因此青藏 高原西北部地区的土壤采样采用挖取土壤剖面的方 式进行, 取样深度分别为 0-10、10-20、20-30和 30-50 cm，同时使用环刀 $\left(100 \mathrm{~cm}^{3}\right)$ 采集 0-10、 10-20、20-30和30-50 cm的土壤样品用于计算不同 层次的土壤容重。此外, 由于机械钻取样无法获得 不同层次土壤容重信息, 我们补充调查了来自 17 个 样地的共计 51 个工程迹地剖面或自然剖面, 用环刀 采集两份土壤样品, 一份用于测定土壤有机碳, 另 一份用于测定容重, 取样深度与机械钻取样深度完 全一致。

\section{3 土壤样品分析}

所有样品带回实验室风干后过 $2 \mathrm{~mm}$ 篎, 去除 $>2 \mathrm{~mm}$ 的石块和根系。土样经球磨机研磨并过 100 目土壤篎后进行样品分析。具体来讲, 我们首先利 用元素分析仪(Vario EL Ш, Elementar, Hanau, Germany)测定土壤全碳含量, 然后采用碳酸盐分析仪 (Calcimeter 08.53, Eijkelkamp, Giesbeek, Netherlands)测定无机碳含量, 二者相减得到有机碳含量。 土壤容重为单位体积土壤在 $105{ }^{\circ} \mathrm{C}$ 条件下的烘干质 量。为了推算基于机械钻获取的土壤样品对应的容 重, 本文建立了土壤容重 $(B D)$ 与有机碳含量 $(S O C)$ 之间的回归关系(公式(1))。

$$
\begin{aligned}
& B D=0.9955+0.5427 \mathrm{e}^{-0.0770 S O C} \\
& \left(R^{2}=0.46, p<0.001\right)
\end{aligned}
$$

需要说明的是, 土壤容重与土壤有机碳含量的 关系是一种较为稳定的经验关系, 这种关系被广泛 用于碳库估算(Avnimelech et al., 2001; Donato et al., 2011; Delvecchia et al., 2014; Hugelius et al., 2014)。
本研究参照前人的做法, 利用工程迹地剖面或自然 剖面的样品建立以土壤容重为因变量, 以土壤有机 碳含量为自变量的回归方程, 来推算机械钻所取样 品的容重大小(Post et al., 1982; Yang et al., 2007, 2009; Zhang et al., 2010; Deng et al., 2014)。在此基 础上, 特定深度的土壤无机碳密度采用公式(2)进行 计算。

$$
S I C D=\sum_{i=1}^{n} T_{i} \times B D_{i} \times S I C_{i} \times \frac{\left(1-C_{i}\right)}{100}
$$

式中: $S I C D 、 T_{i} 、 B D_{i} 、 S I C_{i}$ 和 $C_{i}$ 分别表示土壤无机 碳密度 $\left(\mathrm{kg} \mathrm{C} \cdot \mathrm{m}^{-2}\right)$ 、土壤厚度 $(\mathrm{cm})$ 、容重 $\left(\mathrm{g} \cdot \mathrm{cm}^{-3}\right)$ 、无 机碳含量 $\left(\mathrm{g}^{\mathrm{kg}} \mathrm{kg}^{-1}\right)$ 和 $>2 \mathrm{~mm}$ 的砾石含量 $(\%)$ 。

\section{4 无机碳库估算}

土壤无机碳库的估算分以下3步进行。首先, 基 于 114 个样地中 342 个 $3 \mathrm{~m}$ 深度的土壤剖面信息, 建 立0-100、0-200和0-300 cm 土壤无机碳密度与 0-50 $\mathrm{cm}$ 土壤无机碳密度的关系(表1)。然后，根据表 1 的 关系推算得到研究区西部177个土壤剖面在0-100、 0-200和0-300 cm深度的土壤无机碳密度。最后, 利 用克里格插值得到青藏高原高寒草地 $0-50 、 0-100$ 、 0-200和0-300 $\mathrm{cm}$ 土壤无机碳密度的空间分布, 并 将土壤无机碳密度分布图与青藏高原植被图叠加, 得到不同草地类型的土壤无机碳密度。

表1 0-100、0-200和0-300 $\mathrm{cm}$ 深度土壤无机碳密度与 $0-50 \mathrm{~cm}$ 深度土壤 无机碳密度(SICD)的关系

Table 1 Relationships of soil inorganic carbon density (SICD) at depths of $0-100,0-200$ and $0-300 \mathrm{~cm}$ with SICD in the top $50 \mathrm{~cm}$

\begin{tabular}{lccc}
\hline 方程 Equation & $R^{2}$ & $p$ & $n$ \\
\hline SICD $_{0-100}=1.9309$ SICD $_{0-50}+1.6766$ & 0.90 & $<0.01$ & 114 \\
$S I C D_{0-200}=3.4592$ SICD $_{0-50}+6.8517$ & 0.69 & $<0.01$ & 114 \\
SICD $_{0-300}=4.9222$ SICD $_{0-50}+11.4026$ & 0.61 & $<0.01$ & 114 \\
\hline
\end{tabular}

克里格插值又称空间局部插值法, 是以变异函 数理论和结构分析为基础, 在有限区域内对区域化 变量进行无偏最优估计的一种方法(Krige, 1951; 王 艳妮等, 2008)。克里格插值不仅考虑待估样点与邻 近已知样点的空间位置, 而且还考虑了各邻近样点 间的位置关系, 以及已知数据点的空间相关性。本 研究中土壤无机碳密度的空间插值分析步骤如下: (1)利用QQ图对样点数据进行正态性检验, 对不服 从正态分布的数据采用Box-Cox方式进行转换; (2) 选取最优的半变异函数拟合模型并选用合适的插值 
方法。本研究中 J-bessel为最优半变异函数拟合模型, 空间插值选用普通克里格方法; (3)利用ArcGIS 10.0 地理统计分析模块进行土壤无机碳密度的空间插 值。本研究中所有插值分析及空间制图均在地理信 息系统软件ArcGIS 10.0中完成。

\section{2 研究结果}

2.1 青藏高原高寒草地土壤无机碳密度的垂直分 布特征

图1显示的是土壤碳密度在 $3 \mathrm{~m}$ 深度的垂直分 布特征。从图1可以看出, 高寒草原不同层次的土壤 无机碳密度均显著大于高寒草甸。对高寒草原而言, 随着土壤深度的增加, 土壤无机碳密度并没有呈现 出显著的增加或降低趋势, 且各层次间土壤无机碳 密度差异很小。在高寒草甸, 0-50、50-100、100-150、 150-200、200-250和250-300 cm 土壤无机碳密度的 中位值分别为 $3.26 、 5.00 、 4.98 、 5.57 、 4.81$ 和 $4.60 \mathrm{~kg}$ $\mathrm{C} \cdot \mathrm{m}^{-2}$ 。虽然各层间的差异不显著, 但从数值上看, 0-50 cm 土壤无机碳密度低于其他各层(图1A、1B)。

就各个层次土壤无机碳密度占总量的比例而 言, 高寒草原0-50和50-100 $\mathrm{cm}$ 深度在总无机碳中
所占的比例显著大于高寒草甸, 而其他层次的比例 分布在两种草地类型间无显著差异。在高寒草原中, 随着深度的增加, 不同层次所占的比例呈显著下降 的趋势，0-50和50-100 $\mathrm{cm}$ 土壤无机碳所占比例最 高。但对高寒草甸而言, 0-50 cm 土壤无机碳所占比 例的中位值为 $9.78 \%$ ，显著低于其他各个层次，而 100-150 $\mathrm{cm}$ 土壤无机碳所占比例最高(中位值为 17.92\%)(图1C、1D)。

\section{2 青藏高原高寒草地土壤无机碳库及空间分布} 特征

青藏高原高寒草地 $0-50 、 0-100 、 0-200$ 和 $0-300$ $\mathrm{cm}$ 土壤无机碳密度分别为 $7.22 、 15.58 、 31.76$ 和 47.46 $\mathrm{kg} \mathrm{C} \cdot \mathrm{m}^{-2}$, 对应的土壤无机碳库分别为 $8.26 、 17.82$ 、 36.33 和54.29 Pg C。高寒草原土壤无机碳库远远大 于高寒草甸, 在不同深度高寒草原土壤无机碳库占 高寒草地总碳库的 $63 \%-66 \%$ 。深层土壤无机碳所占 比例很大, $1 \mathrm{~m}$ 以下土壤无机碳库约为 $1 \mathrm{~m}$ 以内土壤 无机碳库的2倍(表2)。

青藏高原高寒草地不同深度土壤无机碳密度的 空间变异较大。0-50、0-100、0-200和0-300 $\mathrm{cm}$ 土 壤无机碳密度范围分别为 $0-15.88 、 0-32.32 、 3.79-$
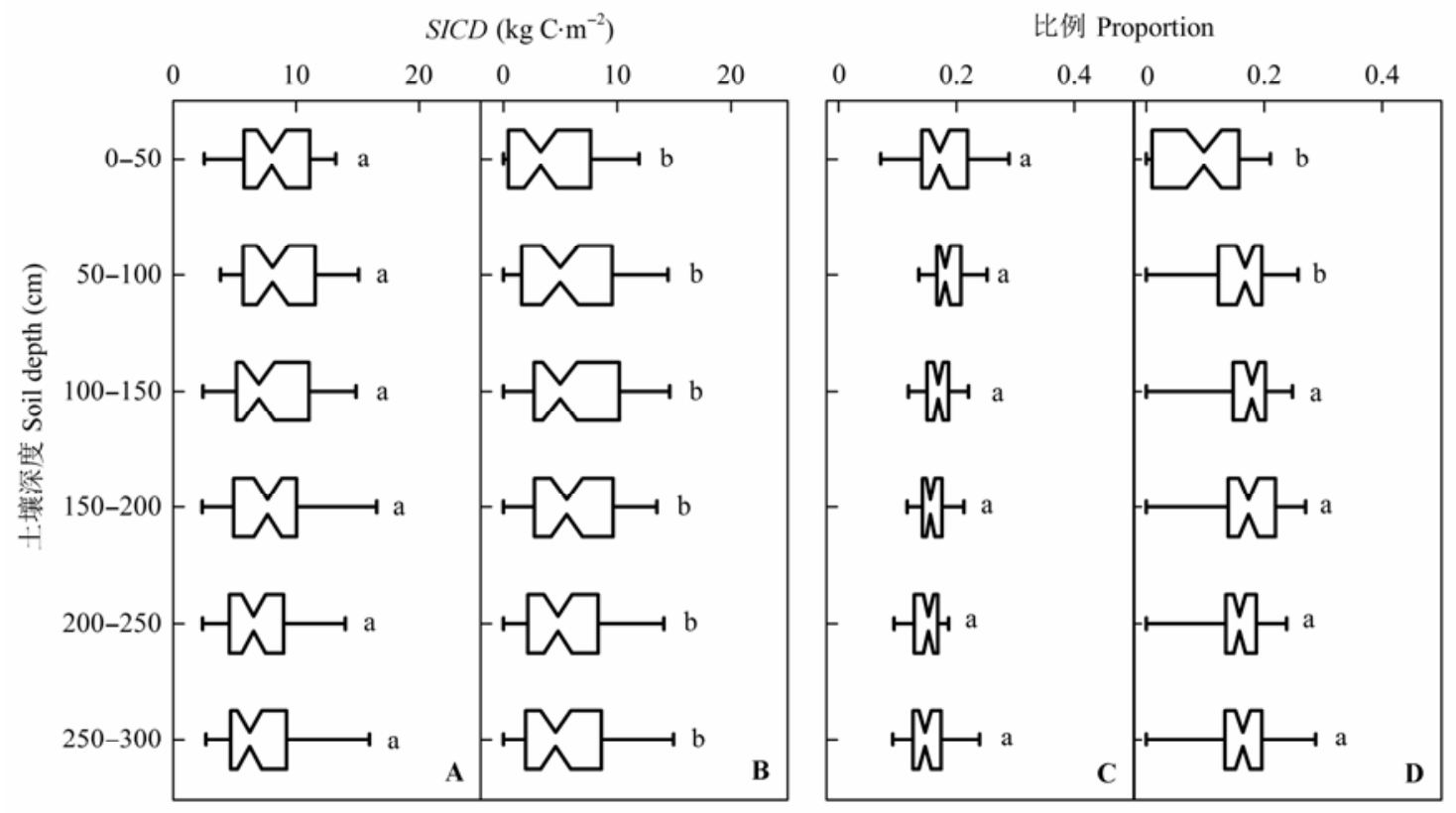

图1 青藏高原高寒草地 $3 \mathrm{~m}$ 深度土壤无机碳密度 $(S I C D)$ 及各层在 $3 \mathrm{~m}$ 深度无机碳密度中所占比例的垂直分布特征。A, 高寒草 原各层土壤无机碳密度。 $\mathbf{B}$, 高寒草甸各层土壤无机碳密度。C, 高寒草原各层土壤无机碳密度在0-300 $\mathrm{cm}$ 无机碳密度中所占 比例。 D, 高寒草甸各层土壤无机碳密度在0-300 $\mathrm{cm}$ 无机碳密度中所占比例。图中字母 $\mathrm{a} 、 \mathrm{~b}$ 表示两种草地类型之间不同层次 土壤无机碳密度差异的显著性检验结果(检验方法为Kruskal-Wallis检验，显著性水平为0.05)。

Fig. 1 Vertical distributions of soil inorganic carbon density $(S I C D)$ at $50 \mathrm{~cm}$ intervals and relative proportion of each layer to total SICD in the 3 meters for alpine steppe $(\mathbf{A}, \mathbf{C})$ and alpine meadow $(\mathbf{B}, \mathbf{D})$. Letters indicate significant differences between the two grasslands types at each depth interval (Kruskal-Wallis test, $p<0.05$ ). 
表2 青藏高原高寒草地不同深度土壤无机碳密度(SICD)和碳库

Table 2 Summary of estimated soil inorganic carbon density (SICD) and soil inorganic carbon (SIC) stock in alpine grasslands on the Tibetan Plateau

\begin{tabular}{|c|c|c|c|c|c|c|c|c|c|}
\hline \multirow{2}{*}{$\begin{array}{l}\text { 草地类型 } \\
\text { Grassland types }\end{array}$} & \multirow{2}{*}{$\begin{array}{l}\text { 面积 Area } \\
\left(10^{4} \mathrm{~km}^{2}\right)\end{array}$} & \multicolumn{4}{|c|}{$\operatorname{SICD}\left(\mathrm{kg} \mathrm{C} \cdot \mathrm{m}^{-2}\right)$} & \multicolumn{4}{|c|}{ 无机碳库 SIC stock (Pg C) } \\
\hline & & $0-50 \mathrm{~cm}$ & $0-100 \mathrm{~cm}$ & $0-200 \mathrm{~cm}$ & $0-300 \mathrm{~cm}$ & $0-50 \mathrm{~cm}$ & $0-100 \mathrm{~cm}$ & $0-200 \mathrm{~cm}$ & $0-300 \mathrm{~cm}$ \\
\hline 高寒草原 Alpine steppe & 64.0 & 8.59 & 18.16 & 36.20 & 53.66 & 5.49 & 11.62 & 23.17 & 34.35 \\
\hline 高寒草甸 Alpine meadow & 50.4 & 5.48 & 12.29 & 26.12 & 39.58 & 2.76 & 6.20 & 13.16 & 19.95 \\
\hline 总计 Total & 114.4 & 7.22 & 15.58 & 31.76 & 47.46 & 8.26 & 17.82 & 36.33 & 54.29 \\
\hline
\end{tabular}

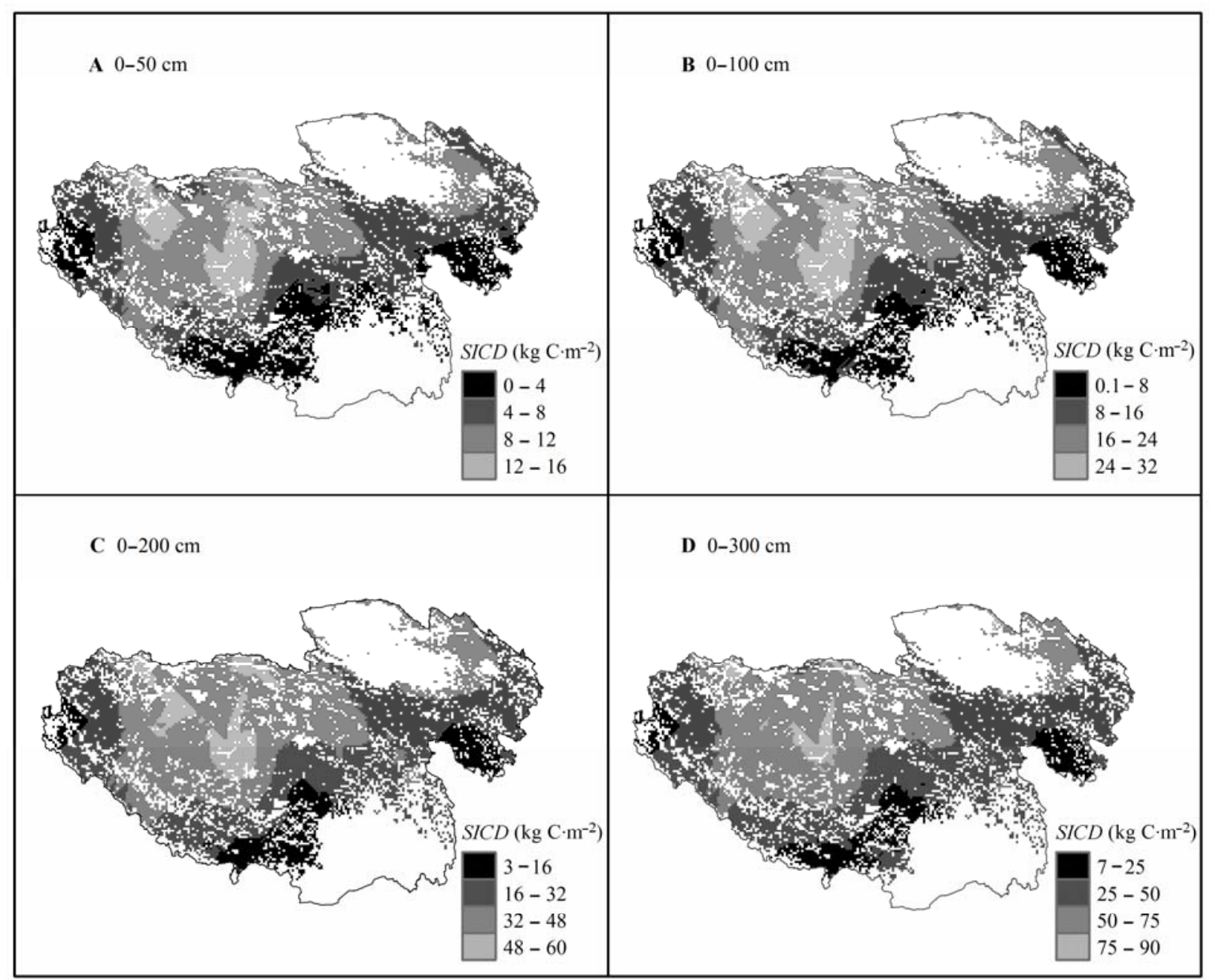

图2 青藏高原高寒草地不同深度土壤无机碳密度 $(S I C D)$ 的空间分布(分辨率为 $10 \mathrm{~km} \times 10 \mathrm{~km}$ )。

Fig. 2 Spatial distributions of estimated soil inorganic carbon density (SICD) at different soil depths across alpine grasslands on the Tibetan Plateau at a resolution of $10 \mathrm{~km} \times 10 \mathrm{~km}$.

60.86和7.02-90.37 $\mathrm{kg} \mathrm{C} \cdot \mathrm{m}^{-2}$ (图2)。即使在同一草地 类型内部, 土壤无机碳密度仍存在较大的变异, 以 0-100 $\mathrm{cm}$ 深度土壤无机碳密度为例, 高寒草原土壤 无机碳密度的范围为 $1.50-32.32 \mathrm{~kg} \mathrm{C} \cdot \mathrm{m}^{-2}$, 高寒草 甸土壤无机碳密度的范围为 $0-28.79 \mathrm{~kg} \mathrm{C} \cdot \mathrm{m}^{-2}$ 。总体 上, 不同深度土壤无机碳密度的空间分布呈现由西 北向东南递减的特点(图2), 土壤无机碳密度最高的 区域分布于西藏自治区北部。

\section{3 讨论}

\section{1 青藏高原高寒草地土壤无机碳库}

青藏高原 $1 \mathrm{~m}$ 深度的总无机碳库为 $17.82 \mathrm{Pg}$, 平 均碳密度为 $15.58 \mathrm{~kg} \mathrm{C} \cdot \mathrm{m}^{-2}$ (表2)。这与前人对该地区 中的估算存在一定差异。具体来讲，Yang等(2010) 估算的青藏高原高寒草地 $1 \mathrm{~m}$ 深度的总无机碳库为 $15.19 \mathrm{Pg}$, 平均碳密度为 $13.46 \mathrm{~kg} \mathrm{C} \cdot \mathrm{m}^{-2}$, 稍低于我 
们的估算结果。这种差异可能是由不同的测试方法 造成的。Yang等(2010)的研究中土壤无机碳含量是 采用总碳与有机碳含量相减得到的, 而本研究中土 壤无机碳含量则是利用碳酸盐分析仪直接测定得 到的。

青藏高原高寒草地仅占我国国土面积的 $12 \%$, 但其 $3 \mathrm{~m}$ 深度土壤无机碳库为 $54.29 \mathrm{Pg} \mathrm{C}$ (表2), 相 当于我国目前估计的土壤无机碳库的 $70 \%$ 左右(潘 根兴, 1999; Li et al., 2007; Wu et al., 2009), 约为世 界土壤无机碳库的 $6 \%$ (Schlesinger, 1982)。因此, 青 藏高原高寒草地是我国极为重要的土壤无机碳库, 也是全球土壤无机碳库的重要组成部分。此外, 青 藏高原高寒草地 $1 \mathrm{~m}$ 深度土壤无机碳库为同等深度 土壤有机碳库的2倍左右(Yang et al., 2008), 3 m深度 土壤无机碳库是同等深度土壤有机碳库的3倍左右 (Ding et al., 2016), 这意味着青藏高原区域碳估算 中不能忽视土壤无机碳。

青藏高原高寒草地 $1 \mathrm{~m}$ 以下土壤无机碳库是 1 $\mathrm{m}$ 以内土壤无机碳库的 2 倍左右, 表明青藏高原深 层土壤无机碳所占比例很大。和本研究的结果类似, 西班牙Guadix-Baza盆地2-3 $\mathrm{m}$ 无机碳库为 $2 \mathrm{~m}$ 以上 无机碳库的两倍以上(Díaz-Hernández, 2010), 兰州 黄土高原 1-2 $\mathrm{m}$ 无机碳库与 0-1 $\mathrm{m}$ 无机碳库基本相 同(Zhang et al., 2015), Wang等(2010)对5种生态系 统土壤中无机碳含量的研究发现沙漠中 1-3 m 深度 土壤碳主要以无机碳形式存在。从这些研究中可以 看出, 深层土壤中储存着大量无机碳。如果忽视这 部分无机碳, 将严重低估土壤中无机碳库大小。因 此, 深层土壤无机碳应该在未来碳循环研究中予以 重视。

需要说明的是, 由于青藏高原西部恶劣的道路 条件所限, 使得深层采样工作无法在西部开展, 从 而导致我们的深层采样点主要集中在青藏高原东部 区域。考虑到青藏高原东西部的土壤母质和降水条 件存在差异, 这两个区域土壤深、浅层无机碳密度 的关系可能存在差异, 进而给青藏高原西部深层无 机碳库的估算带来一定的不确定性。为了检验基于 青藏高原东部区域0-100、0-200和0-300 cm深度的 土壤无机碳密度与 0-50 $\mathrm{cm}$ 土壤无机碳密度的关系 推导西部区域深层土壤无机碳库的可靠性, 我们利 用青藏高原东部采样点 $0-30 \mathrm{~cm}$ 的土壤无机碳密度 $\left(S I C D_{0-30 \mathrm{~cm}}\right)$ 和0-50 $\mathrm{cm}$ 的土壤无机碳密度 $\left(S I C D_{0-50 \mathrm{~cm}}\right)$
建立线性回归关系 $\left(S I C D_{0-50 \mathrm{~cm}}=1.7407 S I C D_{0-30 \mathrm{~cm}}+\right.$ $0.5068)$, 然后利用青藏高原西部实测值 $\left(S I C D_{0-50 \mathrm{~cm}}\right)$ 进行了验证。结果表明, 利用高原东部采样点建立 的深、浅层无机碳密度关系在高原西部依然有很好 的预测表现(图3)。这一结果证明青藏高原东西部深 浅层无机碳密度关系较为稳定, 但在将来的研究中 仍需使用深层土壤观测数据来进一步验证。

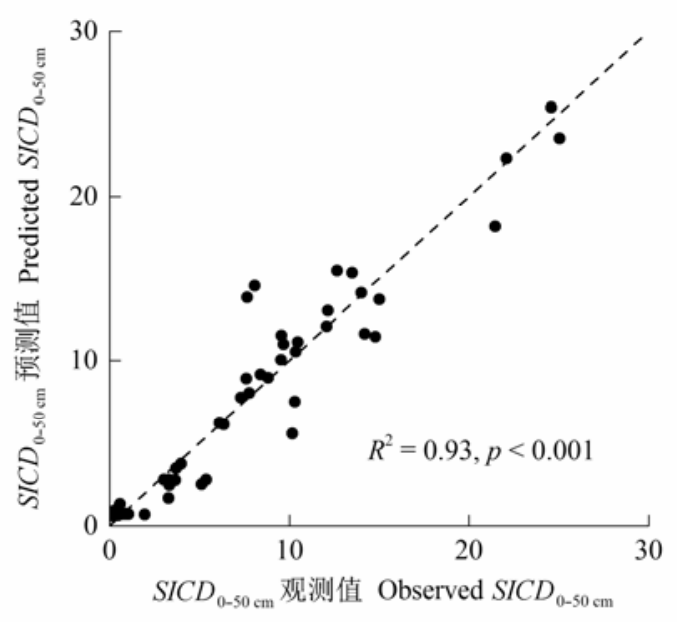

图3 0-50 cm 无机碳密度的预测值与实测值之间的比较。根 据青藏高原东部样点建立的回归关系 $\left(S I C D_{0-50 \mathrm{~cm}}=\right.$ $\left.1.7407 S I C D_{0-30 \mathrm{~cm}}+0.5068\right)$ 在青藏高原西部的预测表现。 $S I C D$, 土壤无机碳密度; 虚线为 $1: 1$ 线。

Fig. 3 Validation of the regression model of soil inorganic carbon density (SICD) between the depths of 0-30 cm and 0-50 $\mathrm{cm}\left(S I C D_{0-50 \mathrm{~cm}}=1.7407 S I C D_{0-30 \mathrm{~cm}}+0.5068\right)$ constructed in the eastern Tibetan Plateau, using actual measurements in the western part of the plateau. The dashed line is 1:1 line.

\section{2 青藏高原土壤无机碳密度的分布特征}

高寒草原的土壤无机碳密度大于高寒草甸中的 观测值, 这可能是由土壤母质的差异所造成的。高 寒草原的土壤母质中含有石灰岩层, 因此与高寒草 甸相比, 高寒草原具有更大的无机碳密度(Yang et al., 2010)。

高寒草地土壤无机碳垂直分布主要表现为高寒 草原不同层次土壤无机碳密度所占比例随着深度的 增加呈显著下降的趋势, 而高寒草甸0-50 $\mathrm{cm}$ 土壤 无机碳密度所占比例显著低于其他各个层次, 100-150 cm 土壤无机碳所占比例最高。近期的许多 研究中都出现了类似的格局(Díaz-Hernández et al., 2003; Díaz-Hernández, 2010; Wang et al., 2010; 刘 淑丽等, 2014)。这种格局可能是由于不同草地类型 
中降水量的差异引起的土壤无机碳的淋溶作用的大 小不同所造成的。已有研究表明, 土壤无机碳随水 分的垂直迁移是土壤无机碳分布格局的一个重要成 因(Wang et al., 2010; Tan et al., 2014), 即无机碳随 土壤溶液向下淋溶迁移的过程中, 随着温度逐渐降 低, $\mathrm{CO}_{2}$ 的溶解度随之降低(杨黎芳和李贵桐, 2011), 因此使得溶液中的无机碳析出形成发生性碳酸盐, 从而导致表层土壤碳含量降低, 而在深层土壤中呈 现土壤无机碳的富集。有意思的是, 在本研究中, 无 机碳所富集的土层深度在两种草地类型之间有差 异。高寒草甸的年降水量较大, 大量的降水导致无 机碳向下迁移, 故表层土壤中无机碳密度较小(刘 淑丽等, 2014), 而在100-150 cm深度土壤无机碳出 现富集。对高寒草原而言, 其年降水量相对较小, 淋 溶作用也小, 因此0-50 $\mathrm{cm}$ 土壤无机碳所占比例最 大(刘淑丽等, 2014)。

总之, 青藏高原土壤无机碳密度的分布特征在 不同草地类型间的差异本质上并不是由植被类型本 身的差异所造成, 而是不同草地类型之间气候条件 和土壤属性差异的综合体现。

\section{4 结论与展望}

青藏高原高寒草地 $3 \mathrm{~m}$ 深度土壤无机碳库为 $54.29 \mathrm{Pg} \mathrm{C}$, 占我国目前估计的土壤无机碳库的 $70 \%$ 左右, 是我国极为重要的土壤无机碳库。其中, $1 \mathrm{~m}$ 以下土壤无机碳库为 $36.47 \mathrm{Pg} \mathrm{C}$, 是 $1 \mathrm{~m}$ 深度土 壤无机碳库的 2 倍, 如果忽略深层土壤中的这部分 无机碳, 将严重低估区域土壤无机碳库大小。因此, 未来碳循环研究中应该对深层土壤无机碳予以更多 关注。

\section{基金项目 国家自然科学基金(31322011和 41371213)。}

致谢 本文得到内蒙古工业大学重点基金(ZD201217)支持, 特此致谢! 感谢青海省农牧厅和林业 厅、西藏自治区农牧厅和林业厅, 以及青海和西藏 各级地方农牧部门和林业部门在野外考察工作中给 予的大力帮助, 同时也感谢中国科学院植物研究所 野外考察队成员帮助采集土壤样品。

\section{参考文献}

Avnimelech Y, Ritvo G, Meijer LE, Kochba M (2001). Water content, organic carbon and dry bulk density in flooded sediments. Aquacultural Engineering, 25, 25-33.

Batjes NH (1996). Total carbon and nitrogen in the soils of the world. European Journal of Soil Science, 47, 151-163.

Delvecchia AG, Bruno JF, Benninger L, Alperin M, Banerjee O, Morales JDD (2014). Organic carbon inventories in natural and restored Ecuadorian mangrove forests. Peerj, 2, doi:10.7717/peerj.388.

Deng L, Shangguan ZP, Sweeney S (2014). "Grain for Green" driven land use change and carbon sequestration on the Loess Plateau, China. Scientific Reports, 4, 7039-7039.

Díaz-Hernández JL (2010). Is soil carbon storage underestimated? Chemosphere, 80, 346-349.

Díaz-Hernández JL, Fernández EB, González JL (2003). Organic and inorganic carbon in soils of semiarid regions: A case study from the Guadix-Baza Basin (Southeast Spain). Geoderma, 114, 65-80.

Ding JZ, Li F, Yang GB, Chen LY, Zhang BB, Liu L, Fang K, Qin SQ, Chen YL, Peng YF, Ji CJ, He HL, Smith P, Yang YH (2016). The permafrost carbon inventory of the Tibetan Plateau: A new evaluation using deep sediment cores. Global Change Biology, doi: 10.1111/gcb.13257.

Donato DC, Kauffman JB, Murdiyarso D, Kurnianto S, Stidham M, Kanninen M (2011). Mangroves among the most carbon-rich forests in the tropics. Nature Geoscience, 4, 293-297.

Georg W, Fenstermaker LF, Arnone IJA (2008). Large annual net ecosystem $\mathrm{CO}_{2}$ uptake of a Mojave Desert ecosystem. Global Change Biology, 14, 1475-1487.

Harrison EJ, Dorn RI (2014). Introducing a terrestrial carbon pool in warm desert bedrock mountains, southwestern USA. Global Biogeochemical Cycles, 28, 253-268.

Hugelius G, Strauss J, Zubrzycki S, Harden JW, Schuur EAG, Ping CL, Schirrmeister L, Grosse G, Michaelson GJ, Koven CD (2014). Estimated stocks of circumpolar permafrost carbon with quantified uncertainty ranges and identified data gaps. Biogeosciences, 11, 6573-6593.

Jacobson AD, Blum JD, Walter LM (2002). Reconciling the elemental and $\mathrm{Sr}$ isotope composition of Himalayan weathering fluxes: Insights from the carbonate geochemistry of stream waters. Geochimica et Cosmochimica Acta, 66, 3417-3429.

Krige D (1951). A statistical approach to some basic mine valuation problems on the Witwatersrand. Journal of Chemical, Metallurgical, and Mining Society of South Africa, 52, 119-139.

Lal R (2004). Soil carbon sequestration impacts on global climate change and food security. Science, 304, 1623-1627.

Landi A, Mermut AR, Anderson DW (2003). Origin and rate of pedogenic carbonate accumulation in Saskatchewan soils, Canada. Geoderma, 117, 143-156.

Lerman A, Mackenzie FT (2005). $\mathrm{CO}_{2}$ air-sea exchange due to calcium carbonate and organic matter storage, and its implications for the global carbon cycle. Aquatic Geochemistry, 11, 345-390.

Li MS (1994). The soil in Tibet. China's Tibet, 3, 29-31. (in 
Chinese) [李明森 (1994). 西藏的土壤. 中国西藏, 3, 29-31.]

Li ZP, Han FX, Su Y, Zhang TL, Sun B, Monts DL, Plodinec MJ (2007). Assessment of soil organic and carbonate carbon storage in China. Geoderma, 138, 119-126.

Liu SL, Lin L, Guo XW, Li J, Ouyang JZ, Du YG, Zhang FW, Li YK, Cao GM (2014). The variation feature of soil inorganic carbon storage in alpine grassland in Qinghai Province. Acta Ecologica Sinica, 34, 5953-5961. (in Chinese with English abstract) [刘淑丽, 林丽, 郭小伟, 李婧, 欧 阳经政, 杜岩功, 张法伟, 李以康, 曹广民 (2014). 青 海省高寒草地土壤无机碳储量空间分异特征. 生态学 报, 34, 5953-5961.]

Liu Z, Dreybrodt W, Liu H (2011). Atmospheric $\mathrm{CO}_{2}$ sink: Silicate weathering or carbonate weathering? Applied Geochemistry, 26, S292-S294.

Liu Z, Dreybrodt W, Wang H (2010). A new direction in effecttive accounting for the atmospheric $\mathrm{CO}_{2}$ budget: Considering the combined action of carbonate dissolution, the global water cycle and photosynthetic uptake of DIC by aquatic organisms. Earth-Science Reviews, 99, 162-172.

Mi N, Wang S, Liu J, Yu G, Zhang W, Jobbagy E (2008). Soil inorganic carbon storage pattern in China. Global Change Biology, 14, 2380-2387.

Mikhailova EA, Post CJ (2006). Effects of land use on soil inorganic carbon stocks in the Russian Chernozem. Journal of Environmental Quality, 35, 1384-1388.

Pan GX (1999). Study on carbon reservoir in soils of China. Bulletin of Science and Technology, 15, 330-332. (in Chinese with English abstract) [潘根兴 (1999). 中国土壤有 机碳和无机碳库量研究. 科技通报, 15, 330-332.]

Post WM, Emanuel WR, Zinke PJ, Stangenberger AG (1982). Soil carbon pools and world life zones. Nature, 298, 156-159.

Schlesinger WH (1982). Carbon storage in the caliche of arid soils: A case study from Arizona. Soil Science, 133, 247-255.

Somebroek W (1993). Amounts, dynamics and sequestering of carbon in tropical and subtropical soils. Ambio, 22, $417-426$.

Stone R (2008). Have desert researchers discovered a hidden loop in the carbon cycle? Science, 320, 1409-1410.

Tan WF, Zhang R, Cao H, Huang CQ, Yang QK, Wang MK, Koopal LK (2014). Soil inorganic carbon stock under different soil types and land uses on the Loess Plateau region of China. Catena, 121, 22-30.

Wang HR, Yang ZF (2011). Research progress on soil inorganic carbon. Journal of Anhui Agriculture Science, 39, 21735-21739. (in Chinese with English abstract) [王海荣, 杨忠芳 (2011). 土壤无机碳研究进展. 安徽农业科学, 39, 21735-21739.]

Wang Y, Li Y, Ye X, Chu Y, Wang X (2010). Profile storage of organic/inorganic carbon in soil: From forest to desert. Science Total Environment, 408, 1925-1931.

Wang YN, Xie JM, Guo X (2008). Application of geostatistical interpolation method in ArcGIS. Software Guide, 12, 36-38. (in Chinese with English abstract) [王艳妮, 谢金 梅, 郭祥 (2008). ArcGIS中的地统计克里格插值法及其 应用. 软件导刊, 12, 36-38.]

Wu H, Guo Z, Gao Q, Peng C (2009). Distribution of soil inorganic carbon storage and its changes due to agricultural land use activity in China. Agriculture, Ecosystems \& Environment, 129, 413-421.

Xu NZ, Zhang TL, Wang XX, Liu HY, Liang XH (2009). Statistical calculation of soil inorganic carbon stock in the Yangtze Delta region. Resources and Environment in the Yangtze Basin, 11, 1038-1044. (in Chinese with English abstract) [许乃政, 张桃林, 王兴祥, 刘红樱, 梁晓红 (2009). 长江三角洲地区土壤无机碳库研究. 长江流域 资源与环境, 11, 1038-1044.]

Yang LF, Li GT (2011). Advances in research of soil inorganic carbon. Chinese Journal of Soil Science, 4, 986-990. (in Chinese with English abstract) [杨黎芳, 李贵桐 (2011). 土壤无机碳研究进展. 土壤通报, 4, 986-990.]

Yang LF, Li GT, Li BG (2006). Modeling and application of stable carbon isotope of pedogenic carbonate. Advances in Earth Science, 9, 973-981. (in Chinese with English abstract) [杨黎芳, 李贵桐, 李保国 (2006). 土壤发生性碳 酸盐碳稳定性同位素模型及其应用. 地球科学进展，9, 973-981.]

Yang YH, Fang JY, Ji CJ, Ma WH, Su SD, Tang ZY (2010). Soil inorganic carbon stock in the Tibetan alpine grasslands. Global Biogeochemical Cycles, 24, GB4022, doi: 10.1029/2010GB003804.

Yang YH, Fang JY, Smith P, Tang YH, Chen AP, Ji CJ, Hu HF, Rao S, Tan K, He JS (2009). Changes in topsoil carbon stock in the Tibetan grasslands between the 1980s and 2004. Global Change Biology, 15, 2723-2729.

Yang YH, Fang JY, Tang YH, Ji CJ, Zheng CY, He JS, Zhu B (2008). Storage, patterns and controls of soil organic carbon in the Tibetan grasslands. Global Change Biology, 14, 1592-1599.

Yang YH, Ji CJ, Chen LY, Ding JZ, Cheng X, Robinson D (2015). Edaphic rather than climatic controls over ${ }^{13} \mathrm{C}$ enrichment between soil and vegetation in alpine grasslands on the Tibetan Plateau. Functional Ecology, 29, 839-848. doi: 10.1111/1365-2435.12393.

Yang YH, Mohammat A, Feng J, Zhou R, Fang JY (2007). Storage, patterns and environmental controls of soil organic carbon in China. Biogeochemistry, 84, 131-141.

Yu BH, Lü CH (2011). Assessment of ecological vulnerability on the Tibetan Plateau. Geographical Research, 12, 2289-2295. (in Chinese with English abstract) [于伯华, 吕昌河 (2011). 青藏高原高寒区生态脆弱性评价. 地理

www.plant-ecology.com 
研究, 12, 2289-2295.]

Yu J, Fang L, Bian ZF, Wang Q, Yu YC (2014). A review of the composition of soil carbon pool. Acta Ecologica Sinica, 34, 4829-4838. (in Chinese with English abstract) [余健, 房莉, 市正富, 汪青, 俞元春 (2014). 土壤碳库 构成研究进展. 生态学报, 34, 4829-4838.]

Zhang F, Wang X, Guo T, Zhang P, Wang J (2015). Soil organic and inorganic carbon in the loess profiles of Lanzhou area: Implications of deep soils. Catena, 126, 68-74.

Zhang K, Dang H, Tan S, Cheng X, Zhang Q (2010). Change in soil organic carbon following the 'Grain-for-Green' programme in China. Land Degradation \& Development, 21, 13-23.
Zheng JF, Cheng K, Pan GX (2011). Perspectives on studies on soil carbon stocks and the carbon sequestration potential of China. Chinese Science Bulletin, 56, 2162-2173. (in Chinese) [郑聚锋, 程琨, 潘根兴 (2011). 关于中国土壤碳 库及固碳潜力研究的若干问题. 科学通报, 56, 21622173.]

Zhou XM, Wang ZB, Du Q (1986). The Vegetation of Qinghai. Qinghai People's Press, Xining. 17. (in Chinese) [周兴民, 王志彬, 杜庆 (1986). 青海植被. 青海人民出版社, 西 宁. 17.]

责任编委: 孙建新 责任编辑: 王 蒇

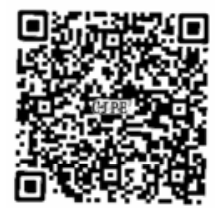

植物生态学报官网

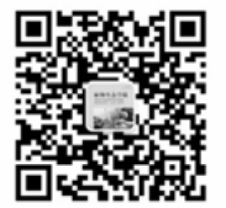

微信订阅号

期刊及学科

相关信息发布

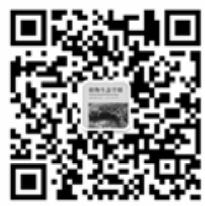

微信服务号

稿件状态查询

全文检索汶览 